\title{
Les incertitudes sur la dose délivrée aux voies respiratoires après exposition au radon
}

\author{
G. MONCHAUX*, R. MASSE**
}

(Manuscrit reçu le 31 juillet 1998, révisé le 28 septembre 1998, accepté le 9 juin 1999)

RESUMÉ L'irradiation des tissus de l'arbre respiratoire par des radioéléments d'origine naturelle provient principalement de l'exposition au radon et à ses descendants à vie courte. La dose délivrée aux différentes régions du poumon après inhalation de radon et de ses descendants ne peut pas être mesurée directement. Elle doit être calculée en utilisant des modèles mathématiques qui prennent en compte les différents processus impliqués, inhalation, dépôt, clairance et décroissance des descendants dans les voies respiratoires. Après un bref rappel historique des principaux modèles dosimétriques, une revue des principaux modèles morphométriques utilisés pour la modélisation est réalisée. Le nouveau modèle de la Commission internationale de protection radiologique (CIPR) est présenté et l'influence de la localisation et de la répartition des cellules cibles, et des paramètres liés au dépôt et à la clairance sur le calcul des doses est décrite. Enfin, une comparaison entre les différents modèles dosimétriques et l'influence des variables de modélisation est réalisée afin d'évaluer les incertitudes sur les calculs des doses.

ABSTRACT Uncertainties on the dose delivered to the respiratory tract after radon exposure.

Irradiation of the different tissues of the respiratory tract by radionuclides of natural origin is mainly related with exposure to radon and its short-lived decay products. The dose to the different regions of the lung arising from inhalation of radon progeny cannot be measured directly. It must be calculated using models which take into account the various processes involved: inhalation, deposition, clearance and decay of radon progeny in the airways of the lung. This paper reviews the main historical dosimetric models and the different morphometric models used for dosimetric purpose. The new Human Respiratory Tract Model for Radiological Protection (ICRP) and the influence of the localisation and distribution of target cells and that of deposition and clearance parameters on dose calculations are described. $A$ comparison between the different dosimetric models and the influence of modelling variables on the uncertainties of dose calculations are addressed.

\footnotetext{
* CEA-DSV-DRR, B.P. 6, 92265 Fontenay-aux-Roses cedex, France.

** CEA-DSV, B.P. 6, 92265 Fontenay-aux-Roses cedex, France.
} 


\section{Introduction}

L'irradiation des tissus de l'arbre respiratoire par des radioéléments d'origine naturelle provient principalement de l'exposition au radon et à ses produits de filiation. Le radon est un gaz noble qui se présente sous trois principales formes isotopiques : ${ }^{222} \mathrm{Rn}$ (radon), ${ }^{220} \mathrm{Rn}$ (thoron) et ${ }^{219} \mathrm{Rn}$ (actinon), formés respectivement lors de la décroissance de ${ }^{238} \mathrm{U},{ }^{232} \mathrm{Th}$ et ${ }^{235} \mathrm{U}$. Du fait de la faible teneur de l'écorce terrestre en ${ }^{235} \mathrm{U}$, la production de ${ }^{219} \mathrm{Rn}$ est très restreinte ; de plus, sa faible période radioactive fait qu'il est pratiquement absent de notre environnement. Le ${ }^{220} \mathrm{Rn}$ est produit en grande quantité, mais sa désintégration rapide fait que les activités volumiques de cet isotope dans l'atmosphère sont le plus souvent faibles. Les risques pour la santé proviennent principalement de l'exposition au ${ }^{222} \mathrm{Rn}$ qui a la demi-vie la plus longue des trois isotopes : 3,824 jours. Le ${ }^{222} \mathrm{Rn}$ est ubiquitaire dans la mesure où il se présente sous la forme d'un radionucléide gazeux, extrêmement mobile et que ses précurseurs, ${ }^{238} \mathrm{U}$ et ${ }^{226} \mathrm{Ra}$ sont présents partout dans l'écorce terrestre. Les produits de filiation du radon sont des radioisotopes de métaux lourds (polonium, plomb, bismuth) qui se présentent sous la forme d'atomes ou d'ions, soit libres, soit adsorbés sur les particules de l'aérosol atmosphérique (Fig. 1). L'aérosol vecteur des produits de filiation du radon est constitué à la fois de particules sur lesquelles sont adsorbés ces produits de filiation et de descendants nouvellement formés qui ne sont pas encore liés à des particules. Comme les autres gaz rares, le radon gaz inhalé, du fait de sa très faible réactivité chimique, n'a que peu d'affinités avec le milieu biologique et est presque entièrement réexhalé. Ses descendants à vie courte qui ont un fort potentiel énergétique se déposent le long des voies aériennes en fonction de leurs paramètres de taille et délivrent leur énergie aux cellules et tissus proches du site de dépôt. Par contre, les descendants à vie longue du ${ }^{222} \mathrm{Rn}$ ainsi que le ${ }^{212} \mathrm{~Pb}$, descendant du ${ }^{220} \mathrm{Rn}$ qui est un émetteur $\beta$ de période 10,6 heures, peuvent être transférés vers le sang, puis par voie systémique, vers les autres organes.

La dose délivrée aux différentes régions du poumon après inhalation de radon et de ses descendants ne peut pas être mesurée directement. Elle doit être calculée en utilisant des modèles mathématiques qui prennent en compte les différents processus impliqués, inhalation, dépôt, clairance et décroissance des descendants dans les voies respiratoires. La dose d'irradiation alpha délivrée aux tissus est fonction : (a) de la concentration des descendants du radon dans l'air, (b) du volume d'air inhalé par le sujet, travailleur ou membre de la population générale, au cours de la période considérée, (c) de la fraction des descendants du radon qui reste déposée dans les différentes régions de l'arbre respiratoire. Par ailleurs, la fraction des descendants du radon déposée dans l'arbre respiratoire est fonction de la géométrie des voies aériennes elles-mêmes, de la fréquence respiratoire, du mode de respiration (par le nez ou par la bouche) et de la taille des particules inhalées. Parmi tous ces paramètres, la seule quantité mesurée habituellement est la concentration des descendants du radon dans l'air. Les autres paramètres, qui dépendent de facteurs environnementaux ou personnels, sont des 


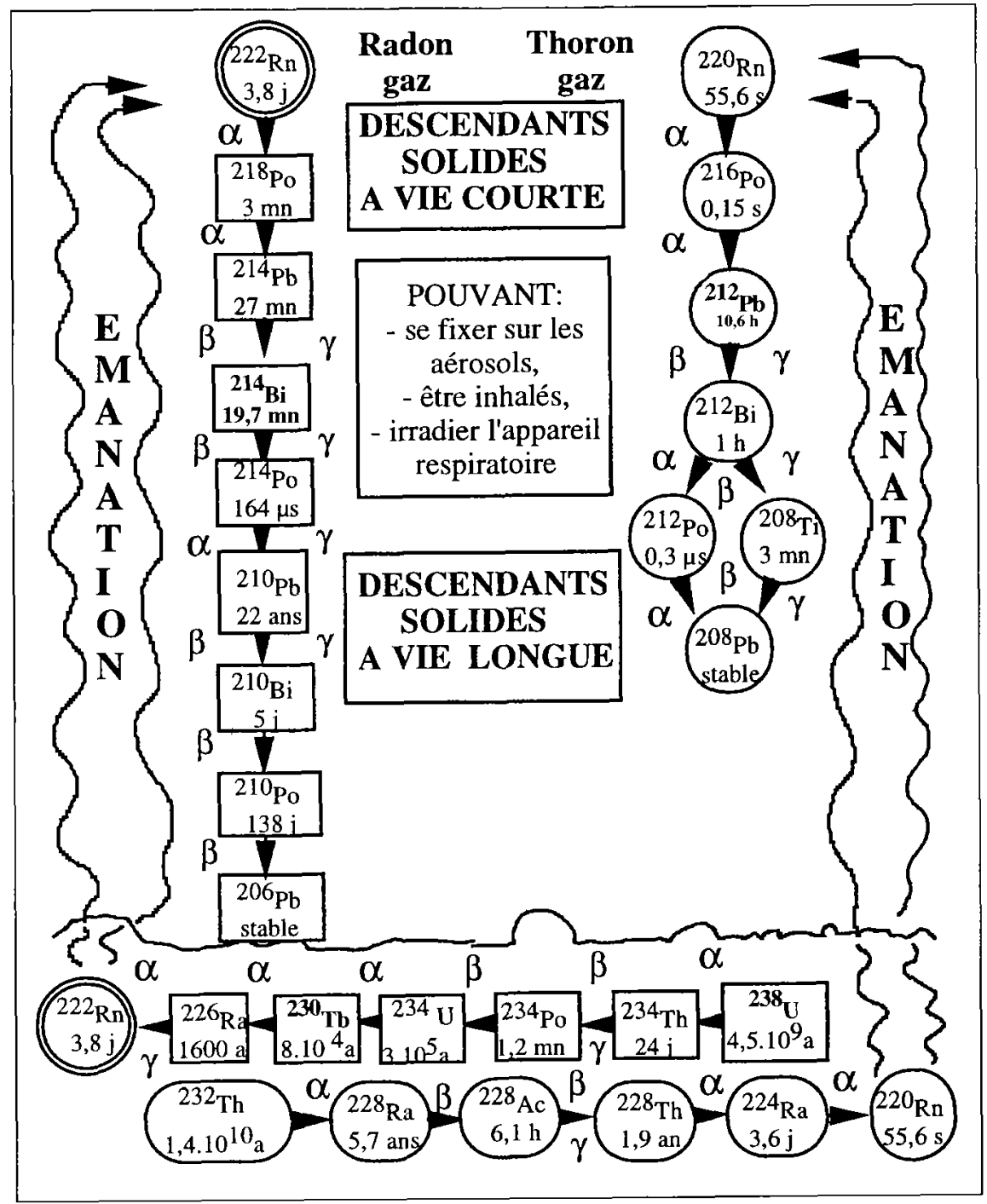

Fig.1. - Filiation radioactive de ${ }^{238} \mathrm{U}$ et ${ }^{232} \mathrm{Th}$ : place des 2 isotopes naturels les plus répandus de l'élément radon.

Radioactive decay of ${ }^{238} \mathrm{U}$ et ${ }^{232} \mathrm{Th}$ : position of the 2 most widespread natural isotopes of the element radon.

valeurs morphométriques moyennes pour la dimension des voies aériennes, la distribution en taille de l'activité inhalée, la fréquence et le mode respiratoires. Ainsi, la dose attribuable à l'inhalation des descendants du radon est toujours 
affectée par des erreurs ou des incertitudes d'importance généralement inconnues. En dépit de ces incertitudes, des efforts ont été accomplis pour quantifier la dose reçue par les poumons après exposition aux descendants du radon.

Les premiers modèles dosimétriques pulmonaires décrivant l'inhalation, le dépôt et la rétention des descendants du radon apparaissent en 1964 (Altschuler et al., 1964; Jacobi, 1964). En 1966, est publié le modèle du Task Group on Lung Dynamics (1966). Le modèle dosimétrique utilisé par la CIPR 30 (ICRP, 1979) est une version légèrement modifiée du modèle publié en 1966. Dans ces deux modèles, la dose calculée est une moyenne rapportée à la masse des poumons remplis de sang permettant de donner une dose moyenne au poumon (concept de dose moyenne au poumon). Dans la publication 30 de la CIPR (ICRP, 1979), la fraction contenue dans les ganglions thoraciques est incluse avec celle contenue dans les poumons. Dans sa publication 32 (ICRP, 1981), la CIPR recommande pour le radon une approche dosimétrique (concept de dose régionale), dans laquelle des doses séparées sont calculées pour la couche basale du tissu bronchique et pour le poumon profond, la moitié du facteur de pondération tissulaire étant appliquée à chacune de ces régions. Ces dispositions ont été maintenues dans la publication 50 (ICRP, 1987). En 1994, dans sa publication 66, la CIPR a publié son nouveau modèle (ICRP, 1994b) qui est une révision majeure du modèle de la CIPR 30 (ICRP, 1979). Enfin, en 1997, aux États-Unis le NCRP a publié son rapport $\mathrm{N}^{\circ} 125$ Deposition, Retention and Dosimetry of Inhaled Radioactive Substances (NCRP, 1997). Il faut cependant signaler que la plupart de ces modèles ont été élaborés afin d'évaluer le détriment radiologique des radionucléides particulaires inhalés, mais ne traitent pas spécifiquement du radon.

\section{Morphométrie}

\section{Modèles morphologiques pulmonaires}

La première étape dans l'évaluation des doses absorbées est de calculer la fraction des descendants du radon déposés à la surface des voies aériennes. Pour ce faire, il est nécessaire d'utiliser un modèle géométrique simplifié de la taille et de la ramification des voies aériennes. Trois principaux modèles ont été utilisés pour la dosimétrie pulmonaire :

\section{Le modèle A de Weibel (Weibel, 1963)}

Ce modèle est basé sur l'étude de coupes histologiques d'un poumon humain et de moulages. Le poumon y est représenté comme une structure unique dans laquelle. chaque voie aérienne se divise de façon dichotomique. Le modèle dosimétrique de Jacobi-Eisfeld (Jacobi and Eisfeld, 1980) utilise le modèle pulmonaire de Weibel. 
INCERTITUDES SUR LA DOSE DÉLIVRÉE AUX VOIES RESPIRATOIRES...

\section{Le modèle de Yeh et Schum (Yeh and Schum, 1980)}

Ce modèle est basé sur l'étude de moulages d'un poumon humain. Dans ce modèle, le poumon est considéré comme un système à cinq lobes dans lequel chaque lobe a ses caractéristiques propres de ramification. Dans cette représentation, les dimensions des voies aériennes sont légèrement plus grandes que celles du modèle de Weibel. Les modèles dosimétriques de James-Birchall (James et al., 1980) et de Harley-Pasternack (Harley and Pasternack, 1982) utilisent le modèle morphométrique de Yeh et Schum ou une forme adaptée de ce modèle.

\section{Le modèle de l'université de Californie à Irvine (UCI) (Phalen et al., 1985)}

Ce modèle est basé sur l'étude d'un grand nombre de moulages de poumon humain provenant de personnes d'âges différents. Les dimensions des voies aériennes sont généralement inférieures à celles données par les modèles de Weibel ou de Yeh et Schum.

Il n'est pas évident de déterminer lequel de ces modèles est le plus adapté pour la dosimétrie pulmonaire. Dans sa publication 66 (ICRP, 1994b), la CIPR a adopté pour les dimensions des voies aériennes dans les régions bronchiques et bronchiolaires des valeurs qui sont des valeurs moyennes pondérées provenant de ces trois modèles.

\section{Le nouveau modèle morphométrique de la CIPR 66 (ICRP, 1994b)}

Le modèle considère l'appareil respiratoire comme étant divisé en quatre régions anatomiques :

(1) la région extrathoracique (ET) qui comprend les voies nasales antérieures (ET $)$ et les régions nasales postérieures, le larynx, le pharynx et la bouche $\left(\mathrm{ET}_{2}\right)$;

(2) la région bronchique (BB) qui comprend la trachée et les bronches dans lesquelles les particules sont épurées par un mécanisme muco-ciliaire;

(3) la région bronchiolaire (bb) qui comprend les bronchioles et les bronchioles terminales, et

(4) la région alvéolaire-interstitielle (AI) qui comprend les bronchioles respiratoires, les canaux et les sacs alvéolaires avec leurs alvéoles et l'interstitium.

Ces quatre régions contiennent des tissus lymphatiques (LT) ou des éléments de ces tissus. Le liquide accumulé dans le tissu interstitiel est recueilli dans les capillaires lymphatiques, les vaisseaux lymphatiques et traverse un ou plusieurs 
ganglions lymphatiques : les ganglions de la région extrathoracique $\left(\mathrm{LN}_{\mathrm{ET}}\right)$ drainent la région extrathoracique et les ganglions thoraciques $\left(\mathrm{LN}_{\mathrm{TH}}\right)$, qui se trouvent dans le territoire bronchique, drainent à la fois ce territoire (BB) et les régions bonchiolaires (bb) et alvéolaires-interstitielles (AI).

Pour les calculs dosimétriques, une voie aérienne est représentée sous la forme d'un tube cylindrique dont le diamètre et l'épaisseur de la paroi sont choisis.

\section{Physiologie respiratoire}

Les doses de radiation délivrées aux tissus et aux cellules de l'arbre respiratoire sont déterminées dans une large mesure par le mode de respiration et par certains paramètres respiratoires dont le plus important est le débit respiratoire. Ces paramètres sont fonction de la taille du sujet et du niveau d'activité physique : sommeil, repos, exercice léger ou travail pénible. Dans les régions extrathoraciques, le mode respiratoire dépend de la résistance relative des voies orales et nasales au flux d'air nécessaire aux besoins ventilatoires. La respiration par le nez est le mode respiratoire prédominant chez les sujets sains au repos. Par contre, dans le cas d'exercice où un fort débit ventilatoire est nécessaire (environ $2,1 \mathrm{~m}^{3} \mathrm{~h}^{-1}$, pour un homme adulte), le mode respiratoire devient oronasal car la bouche offre une résistance moindre au flux aérien. Les variations de la dose en fonction de l'âge des sujets sont liées aux variations du dépôt dans l'arbre respiratoire qui dépend lui-même du débit respiratoire et de la dimension des voies aériennes. Le modèle de la publication 66 (ICRP, 1994b) prévoit peu de différence entre les doses calculées chez l'adulte et l'enfant âgé de plus de 10 ans. Pour les enfants plus jeunes, le modèle indique même des doses plus faibles du fait d'une filtration nasale très importante chez le jeune enfant qui respire presque exclusivement par le nez (Roy et Rannou, 1998).

Pour les calculs dosimétriques, la $C I P R 66$ recommande une série de valeurs de référence pour l'exposition de la population générale d'origine caucasienne. Bien que l'influence de l'ethnie ne soit pas établie, des indications sont fournies pour ajuster ces valeurs pour d'autres groupes ethniques.

\section{Radiobiologie}

La connaissance des types et de la localisation des cancers de l'appareil respiratoire est utile pour identifier les cellules et tissus à risque. 
INCERTITUDES SUR LA DOSE DÉLIVRÉE AUX VOIES RESPIRATOIRES...

\section{Cancers de l'appareil respiratoire}

\section{Voies aériennes extrathoraciques}

En ce qui concerne le risque de cancers des voies aériennes supérieures, il existe à travers le monde une grande variabilité qui est fonction de l'origine ethnique et du mode de vie. La région $\mathrm{ET}_{1}$ n'est pas considérée comme un tissu à risque, bien que les cancers cutanés de l'aile du nez soient assez fréquents dans la population générale. L'incidence des cancers de la cavité buccale, du pharynx et du larynx est fortement influencée par le tabagisme et la consommation d'alcool. Il n'a pas été retrouvé d'excès de cancers radioinduits des voies aériennes supérieures dans les études épidémiologiques sur les survivants d'Hiroshima et de Nagasaki, ni sur les patients traités pour spondylarthrite ankylosante (Darby et al., 1985). Cependant des cancers des sinus ont été observés après injection intrasinusale de Thorotrast chez des patients (Grampa, 1971), chez les peintres de cadrans lumineux au radium (Rowland, 1994), et expérimentalement après injection intrasinusale de ${ }^{144} \mathrm{Ce}$ (Jasmin et al., 1977).

\section{Voies aériennes thoraciques et poumon}

La plupart des cancers respiratoires sont des cancers intrathoraciques d'origine épithéliale et la majorité des cancers pulmonaires sont d'origine bronchique. La moitié des tumeurs est d'origine proximale et survient dans les grosses bronches, jusqu'au point de division des bronches segmentaires ( $3^{\mathrm{e}}$ génération) et l'autre moitié d'origine plus distale survient dans les bronches segmentaires. D'une manière générale, la fréquence des quatre grandes classes de cancers du poumon peut se résumer de la manière suivante (ICRP, 1994b) : carcinomes épidermoïdes (30 à 50\%), carcinomes à petites cellules (15 à $30 \%)$, adénocarcinomes (10 à $30 \%$ ) et carcinomes à grandes cellules (de moins de $10 \%$ à 20\%). Les carcinomes sont des tumeurs épithéliales malignes qui se développent à partir de l'épithélium des bronches ou des bronchioles. Les adénocarcinomes sont des tumeurs malignes qui se développent à partir d'un épithélium glandulaire de n'importe quelle région de l'appareil respiratoire. Des sarcomes, tumeur d'origine conjonctive, ont été observées expérimentalement, notamment à forte dose, et dans cette catégorie, les angiosarcomes prédominent. Des mésothéliomes, tumeurs pleurales et/ou péritonéales d'origine conjonctive, ont été observées chez des patients ayant reçu du Thorotrast (Andersson et al., 1995).

La fréquence de ces quatre grandes classes de cancers diffère entre les fumeurs et les non-fumeurs. Chez les non-fumeurs, environ $30 \%$ des cancers sont des carcinomes épidermoïdes, $20 \%$ des carcinomes à petites cellules, $45 \%$ des adénocarcinomes et $5 \%$ des carcinomes à grandes cellules. Chez les fumeurs, la proportion des carcinomes épidermoïdes peut atteindre $50 \%$, alors que la proportion des adénocarcinomes peut être de l'ordre de $25 \%$. La quasi-totalité des carcinomes 
à petites cellules et la très grande majorité des carcinomes épidermoïdes ont une localisation proximale. Par contre, les adénocarcinomes et les carcinomes à grandes cellules sont majoritairement distaux.

Il est connu que les carcinomes à petites cellules et les carcinomes épidermoïdes sont très liés à la fumée de cigarettes. Cependant, au cours des vingt dernières années, la prévalence des adénocarcinomes a fortement augmenté en rapport avec la consommation de cigarettes moins irritantes à faibles taux de goudron et de nicotine (Muscat and Wynder, 1995; Thun et al., 1997). En parallèle, chez les mineurs d'uranium ayant de fortes expositions au radon (Saccomanno et al., 1964; 1971 ; Horacek et al., 1977), des taux exceptionnellement élevés de cancers à petites cellules ont été observés, en particulier chez les fumeurs (Saccomanno et al., 1988). Une incidence de $66 \%$ de ce type de tumeur a été observé dans une cohorte de mineurs d'uranium du plateau du Colorado (Yessner, 1981). Toutefois, les cas diagnostiqués le plus récemment dans les cohortes de mineurs du plateau du Colorado ne confirment pas ce taux élevé de cancers à petites cellules (Saccomanno et al., 1996). Les carcinomes épidermoïdes y deviennent prédominants et les carcinomes à petites cellules diminuent progressivement jusqu'à $22 \%$. La fréquence des adénocarcinomes augmente jusqu'à environ $15 \%$ pour les mineurs ayant commencé à travailler au fond entre 20 et 29 ans. Pour les deux types d'exposition, la diminution de l'incidence des carcinomes à petites cellules pourrait être en rapport avec des doses plus faibles, doses d'irradiation plus faibles pour les mineurs et doses plus faibles de goudron et de nicotine pour les fumeurs (Wynder and Muscat, 1995).

\section{Cellules à risque}

Il est vraisemblable que les cellules du poumon, qui sont les cellules cibles des cancers radioinduits, sont celles dont la différenciation n'est pas complètement terminée, mais qui ont encore des capacités de division et de différenciation. Parmi les nombreux types cellulaires de l'épithélium bronchique et bronchiolaire, seules les cellules basales et les cellules sécrétoires à petits grains de sécrétion semblent avoir un potentiel de prolifération. Ces deux types cellulaires sont considérés comme étant probablement à l'origine des cancers du poumon à la fois par le NRC aux États-Unis (NRC, 1990) et par la CIPR (ICRP, 1994b). Les autres cellules bronchiques, comme les cellules à mucus ou les cellules séreuses sont des cellules bien différenciées qui ne sont donc probablement pas impliquées dans le développement des tumeurs.

En ce qui concerne la modélisation pulmonaire, la $C I P R$ fait l'hypothèse que les cibles sensibles sont réparties, pour la région extrathoracique (ET), dans la couche basale (cellules basales) de l'épithélium stratifié extra-thoracique, et pour la région thoracique, 
- dans tout l'épithélium bronchique (cellules sécrétoires et cellules basales) de la région bronchique $(\mathrm{BB})$;

- dans tout l'épithélium bronchiolaire (cellules sécrétoires ou cellules de Clara) de la région $(\mathrm{bb})$;

- uniformément dans tout le tissu alvéolo-interstitiel (cellules endothéliales et pneumocytes II) et les ganglions de la région (AI). Les pneumocytes II couvrent seulement $7 \%$ de la surface alvéolaire, mais sont deux fois plus nombreux que les pneumocytes I. La répartition et les caractéristiques des différentes cellules cibles de la région thoracique sont indiquées dans le tableau I.

\section{TABLEAU I}

Répartition et caractéristiques des cellules cibles dans les voies aériennes thoraciques d'après la publication 66 de la CIPR (ICRP, 1994 b)

Distribution and characteristics of target cells in the thoracic airways from ICRP 66 publication (ICRP, 1994 b)

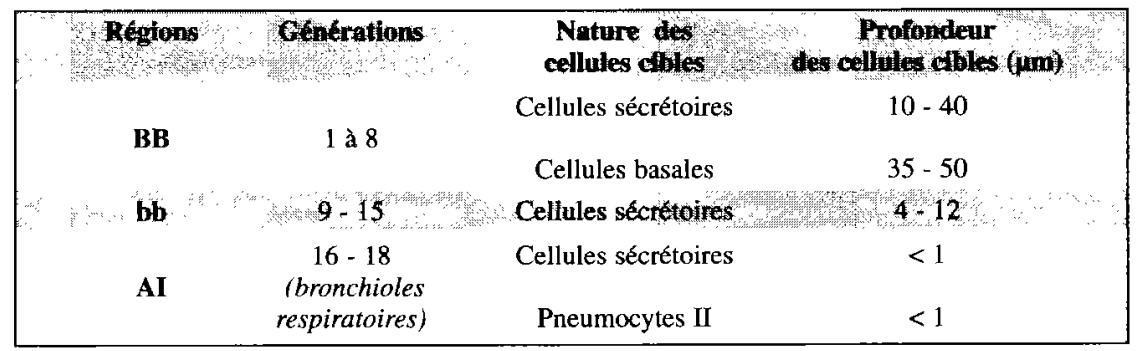

Bien que les cellules basales aient été longtemps considérées comme les cellules souches de l'épithélium des bronches, certains arguments ont été avancés contre le fait que ces cellules soient les principales cellules cibles. Ces arguments sont basés sur le fait que dans les régions les plus distales, l'épithélium bronchique a une très faible densité de cellules basales. Dans certains modèles dosimétriques pulmonaires, tel que le modèle de James-Birchall (James et al., 1980) et dans le nouveau modèle du NCRP (NCRP, 1997), les doses sont calculées à travers toute l'épaisseur de l'épithélium bronchique ce qui équivaut à faire l'hypothèse que les cellules sensibles peuvent se rencontrer dans toute l'épaisseur du tissu. Des données expérimentales suggèrent que les cellules sécrétoires pourraient être les cellules progénitrices les plus importantes pour l'épithélium bronchique et que les cellules basales auraient un pouvoir de différenciation limité (Johnson and Hubbs, 1990). 


\section{Dépôt}

Dans le modèle de la CIPR 66 (ICRP, 1994b), chaque région de l'arbre respiratoire est représentée comme l'équivalent d'un filtre dans lequel les particules sont déposées à l'inspiration comme à l'expiration. Dans ce modèle, une approche semiempirique est utilisée pour décrire les valeurs du dépôt dans les différentes régions à partir des expérimentations et/ou de la théorie selon une approche utilisant la mécanique des fluides.

Dans le cas de l'exposition au radon, la dose délivrée aux tissus de l'appareil respiratoire dépend principalement de la répartition initiale des particules au moment de l'inhalation. La probabilité de dépôt des descendants particulaires dépend:

- de l'inhalabilité de l'aérosol qui est le rapport du nombre des particules ayant un diamètre aérodynamique particulier, inspirées dans l'arbre respiratoire par le nez ou par la bouche, et de la concentration en nombre de particules ayant le même diamètre aérodynamique présentes dans le volume d'air ambiant inspiré. Dans les conditions de l'environnement domestique, la majorité des descendants du radon sont généralement libres ou attachés à des particules de taille submicronique, de sorte que leur inhalabilité est voisine de l'unité ;

- des paramètres respiratoires qui déterminent pour différents groupes de population et pour différentes activités physiques le mode de ventilation (nasal, oral ou oro-nasal) et le débit respiratoire. Le débit respiratoire est une donnée physiologique qui varie pour chaque sujet et pour lequel des valeurs standard moyennes ont été établies : $1,2 \mathrm{~m}^{3} \mathrm{~h}^{-1}$ pour les travailleurs et $0,8 \mathrm{~m}^{3} \mathrm{~h}^{-1}$ pour les adultes du public (UNSCEAR, 1988);

- des mécanismes physiques liés aux caractéristiques granulométriques de l'aérosol. Les probabilités de dépôt total pour une distribution donnée en taille de l'aérosol peut être obtenue en combinant les probabilités tirées des différents mécanismes de dépôt. L'efficacité de dépôt de chaque filtre de l'appareil respiratoire est représentée par deux composantes, l'une, $\eta_{\mathrm{ae}}$, qui intervient dans les processus de dépôt « aérodynamique » d'impaction et de sédimentation gravitationnelle, l'autre, $\eta_{\mathrm{th}}$, qui intervient dans les processus de dépôt « thermodynamique » des particules par diffusion par mouvement brownien. Dans le cas des descendants du radon, les particules ont généralement un diamètre inférieur à $0,5 \mu \mathrm{m}$, et leur diamètre thermodynamique est proche de leur diamètre géométrique de sorte qu'elles sont soumises aux mécanismes thermodynamiques de diffusion.

D'autres phénomènes physiques interviennent dans les mécanismes de dépôt, comme l'attraction électrostatique ou la croissance hygroscopique. En effet, les particules des descendants du radon inhalés peuvent devenir hygroscopiques en arrivant dans les conditions d'humidité saturée des voies aériennes et l'on peut s'attendre à une croissance de leur taille d'environ deux fois celle de la taille originale. 
Les particules se déposent dans les différentes régions anatomiques de l'arbre respiratoire qui se comportent comme une série de filtres à l'inspiration, comme à l'expiration. Ainsi, une fraction des particules sera retenue, l'autre sera réexhalée. Une modélisation du dépôt des particules en fonction de leur diamètre thermodynamique médian en activité (DTMA) a été réalisée pour chaque région anatomique de l' arbre respiratoire. Dans les conditions de l'environnement domestique, il apparaît que le mécanisme de dépôt le plus important dans les régions bronchiques (BB) et bronchiolaires (bb) est le dépôt par diffusion.

\section{Clairance}

La clairance est considérée comme résultant de la compétition entre deux types de mécanismes : d'une part, un transport particulaire qui épure les particules par action mucociliaire vers la gorge où elles sont dégluties, et d'autre part une dissolution et un transfert vers le sang (absorption), qui est supposé être le même pour toutes les régions de l'arbre respiratoire humain. Dans la région alvéolo-interstitielle (AI), les descendants du radon sont déposés sur un mince film de surfactant en contact étroit avec les capillaires pulmonaires.

Pour le calcul des doses, la majorité des modèles fait l'hypothèse que les descendants du radon sont relativement insolubles et sont épurés avec le mucus. Dans tous les modèles, la période d'absorption est longue par comparaison avec la période radioactive des descendants à vie courte du radon (Fig. 1): 3 minutes pour le ${ }^{218}$ Po, 27 minutes pour le ${ }^{214} \mathrm{~Pb}$ et 20 minutes pour le ${ }^{214} \mathrm{Bi}$. Par contre, les descendants à vie longue du ${ }^{222} \mathrm{Rn}$, comme le ${ }^{210} \mathrm{~Pb}$, émetteur $\beta$ qui a une période 22 ans, le ${ }^{210}$ Po émetteur $\alpha$ qui a une période de 138 jours ou le ${ }^{210} \mathrm{Bi}$, émetteur $\beta$ qui a une période de 5 jours peuvent subir des transports de surface dans les voies respiratoires, être adsorbés par les tissus et transférés vers le sang. Il en est de même pour le ${ }^{212} \mathrm{~Pb}$, descendant du ${ }^{220} \mathrm{Rn}$ qui est un émetteur $\beta$ de période 10,6 heures. Après transport par voie systémique, de très petites fractions peuvent atteindre d'autres organes, comme le foie, le rein, le squelette ou la moelle osseuse. Jacobi et Eisfeld ont modélisé l'effet sur la dose de l'absorption sanguine (Jacobi and Eisfeld, 1980). Dans ce modèle, qui s'applique aux conditions d'exposition dans les mines (ventilation, présence d'aérosols : poussières de minerai, fumées de tirs de mines, fumées de disesel, fumée de cigarettes etc.) la « fraction libre » est absorbée rapidement. Toutefois, dans les conditions de la mine, la « fraction libre »n'est que de 1 à $5 \%$ et cette absorption rapide n'affecte pas trop la dose. Récemment, les données publiées à la fin des années 60 à partir d'expérimentations sur des volontaires humains (Booker et al., 1969 ; Hursh and Mercer, 1970 ; Hursh et al., 1969) ont été réévaluées en utilisant le modèle pulmonaire de la CIPR 66 (ICRP, 1994a) afin d'estimer les périodes d'absorption du plomb et du bismuth applicables au ${ }^{214} \mathrm{~Pb}$ et ${ }^{214} \mathrm{Bi}$ des descendants du radon (Marsh and Birchall, 1998a). 
Dans ces études, il a été admis que les descendants du thoron ${ }^{212} \mathrm{~Pb}$ et ${ }^{212} \mathrm{Bi}$ pouvaient être utilisés comme substituts des descendants du radon, respectivement ${ }^{214} \mathrm{~Pb}$ et ${ }^{214} \mathrm{Bi}$ pour la détermination des taux d'absorption sanguine. Les principales conclusions sont que :

- à partir des études sur des volontaires humains, la meilleure estimation pour la période d'absorption sanguine du plomb inhalé sous forme de descendant du radon ou du thoron est de 10 heures avec un intervalle de confiance à $95 \%$ de \pm 2 heures;

- les périodes d'absorption pour la fraction libre et la fraction attachée de ${ }^{212} \mathrm{~Pb}$ sont identiques ;

- les périodes d'absorption du plomb et du bismuth inhalés sous forme de descendants du radon devraient être inférieures à 2 heures pour entraîner une diminution significative de la dose au poumon et cela est extrêmement improbable, au moins pour le ${ }^{214} \mathrm{~Pb}$;

- la période d'absorption pour le bismuth inhalé sous forme de descendant du radon ou du thoron, estimée à partir des études sur des volontaires humains, est d'environ 13 heures. Ce résultat doit cependant être pris avec précaution car il dépend des types de modèles biocinétiques de la CIPR utilisés pour le bismuth et le plomb.

Il est cependant nécessaire d'avoir plus de données expérimentales pour estimer les valeurs des paramètres d'absorption du polonium et du bismuth appropriées pour les descendants du radon.

\section{Dosimétrie}

Le calcul de la dose délivrée à la région alvéolo-interstitielle (AI) est donné par l'expression suivante (James, 1988) :

$$
D_{\mathrm{AI}}=n E_{\mathrm{a}} / m_{\mathrm{AI}}
$$

dans laquelle $n$ est le nombre de décroissances alpha, $E_{\alpha}$ est l'énergie des particules alpha et $m_{\mathrm{AI}}$ la masse de la région alvéolo-interstitielle. Cette expression simple est valide dans la mesure où dans la région alvéolo-interstitielle, les septa interalvéolaires et les parois des capillaires sanguins et lymphatiques sont suffisamment minces pour assurer que les cellules cibles sont distribuées de manière uniforme dans toute la masse du tissu. On peut donc faire l'hypothèse que la dose moyenne reçue par les cellules cibles est la même que celle reçue par la masse totale du tissu.

Le calcul de la dose absorbée dans les régions bronchique (BB) et bronchiolaire (bb) est plus complexe. Des calculs de dose ont été faits en utilisant des niveaux 
INCERTITUDES SUR LA DOSE DÉLIVRÉE AUX VOIES RESPIRATOIRES...

de sophistication variés allant d'une approche extrêmement simple utilisant l'énergie totale déposée dans une masse arbitraire de tissu pulmonaire à ceux dans lesquels sont calculées les doses délivrées à des types cellulaires spécifiques, situés à des profondeurs définies dans chaque type de voie aérienne et prenant en compte les mécanismes de clairance.

Pour la modélisation dosimétrique, l'identification et la distribution spatiale des cellules cibles est d'une importance majeure. L'une des raisons les plus importantes est le fait que la dose délivrée au poumon par les descendants du ${ }^{222} \mathrm{Rn}$ est due entièrement aux descendants à vie courte. En effet, ceux-ci décroissent rapidement après avoir été déposés dans l'arbre respiratoire alors que des radionucléides à demi-vie plus longue ont plus de temps pour être épurés et ne délivrent pas la totalité de leur énergie potentielle, voire aucune, dans le tissu pulmonaire. Dans la mesure où l'énergie des particules alpha est déposée le long du court trajet de la particule dans les tissus ou fluides $(47 \mathrm{~mm}$ et $71 \mathrm{~mm}$, respectivement, pour les alpha du ${ }^{218} \mathrm{Po}$ et du ${ }^{214} \mathrm{Po}$ ), la dose délivrée au tissu pulmonaire provient entièrement des émissions délivrées finalement $\mathrm{par}^{218} \mathrm{Po},{ }^{214} \mathrm{~Pb}$, ${ }^{214} \mathrm{Bi}$ et ${ }^{214} \mathrm{Po}$.

Afin de déterminer la dose reçue par les cellules cibles, on utilise pour les bronches de la région ( $\mathrm{BB}$ ) et les bronchioles de la région (bb) un modèle cylindrique dans lesquels sont représentées les positions des cibles (cellules basales ou sécrétoires) et des sources (descendants du radon en décroissance).

Trois principaux modèles dosimétriques pulmonaires ont été utilisés pour le calcul des doses après inhalation des descendants du radon : le modèle de JacobiEisfeld (Jacobi and Eisfeld, 1980), le modèle de James-Birchall (James et al., 1980) et le modèle de Harley-Pasternack (Harley and Pasternack, 1982), complété par le modèle de Harley (Harley et al., 1996). Dans ces modèles, les principales cellules cibles sont considérées comme étant les cellules basales de l'épithélium bronchique, ce qui n'est pas le cas du modèle de la CIPR 66 (ICRP, 1994b). Les différences les plus importantes entre les modèles résident dans le choix des modèles morphométriques pulmonaires, la profondeur des cellules cibles et les hypothèses concernant le dépôt et la clairance des descendants à vie courte du radon.

Dans le modèle de Harley-Pasternak (Harley and Pasternack, 1982), la clairance se fait uniquement par le mucus et le transfert vers le sang est ignoré. À l'inverse, l'absorption par le sang est prise en compte dans les modèles de JacobiEisfeld (Jacobi and Eisfeld, 1980), et de James-Birchall (James et al., 1980). Dans le modèle de Harley-Pasternak (Harley and Pasternack, 1982), les doses sont calculées à une profondeur fixe de $22 \mu \mathrm{m}$ au-dessous de la surface dans les 10 premières générations bronchiques et à $10 \mu \mathrm{m}$ au-delà de la $10^{\mathrm{e}}$ génération. Dans le récent modèle de Harley (Harley et al., 1996), la dose délivrée aux cellules basales de la région bronchique a été calculée. Des mesures effectuées sur des 
échantillons chirurgicaux de bronche, allant de la $3^{\mathrm{e}}$ à la $6^{\mathrm{e}}$ génération et provenant de plus de 100 personnes ont conduit à déterminer une profondeur moyenne de la couche basale de $27 \mu \mathrm{m}$. Dans ces études, la profondeur de la couche basale était mesurée du milieu du noyau à la surface libre de l'épithélium. À l'inverse, la profondeur variable des cellules cibles est prise en compte dans les modèles de Jacobi-Eisfeld (Jacobi and Eisfeld, 1980) et de James-Birchall (James et al., 1980).

Par ailleurs, dans le cas de l'exposition au radon et à ses descendants, la distribution de l'énergie alpha délivrée aux cellules cibles de l'appareil respiratoire est hétérogène. En utilisant un modèle microdosimétrique statistique, Fischer et al. (1992) ont montré que l'énergie alpha émises par des sources uniformément distribuées de ${ }^{218} \mathrm{Po}(6 \mathrm{MeV})$ et de ${ }^{214} \mathrm{Po} 7,69(\mathrm{MeV})$ était absorbée de façon non homogène par les noyaux des cellules (calcul effectué pour des noyaux cellulaires de $6,5 \mu \mathrm{m}$ de diamètre). Ces auteurs ont montré que pour une dose moyenne de 1 Gy délivrée à l'organe cible, $27 \%$ des noyaux des cellules cibles ne reçoivent aucun dépôt d'énergie et que donc $73 \%$ des cellules recevaient au moins un choc par noyau, avec une dose absorbée moyenne de 0,7 Gy. La dose de $0,5 \mathrm{~Gy}$ correspond à la dose moyenne pour les chocs uniques. La majorité des noyaux des cellules reçoivent des doses inférieures à $2 \mathrm{~Gy}$, mais une petite partie de ces noyaux reçoivent des doses comprises entre 2 et 3,5 Gy. Ces résultats montrent que les interactions par choc unique prédominent dans le cas des faibles expositions alors que les interactions par choc multiple augmentent pour de plus fortes expositions.

\section{La dose efficace par unité d'exposition aux descendants du radon}

Les doses par unité d'exposition à l'énergie alpha potentielle des descendants du radon $P_{\mathrm{p}}$ sont exprimées en terme de dose absorbée $\left(\mathrm{mGy} \mathrm{WLM}^{-1}\right)$. Afin de calculer les doses efficaces $E$ à partir des doses absorbées au poumon, il est nécessaire d'utiliser des facteurs de pondération appropriés qui sont essentiellement :

(a) le facteur de pondération pour l'irradiation alpha $W_{\mathrm{R}}$ (l'ancien facteur de qualité $Q$ ). Pour les particules alpha, la valeur recommandées par la publication 60 de la CIPR (ICRP, 1990) est : $W_{\mathrm{R}}=20$;

(b) un facteur de pondération tissulaire $W_{\mathrm{T}}$. Pour le poumon, la valeur de $W_{\mathrm{T}}$ recommandée par la publication 60 de la CIPR (ICRP, 1990) est de 0,12. Dans son nouveau modèle, la CIPR 66 (ICRP, 1994b) recommande que des facteurs de pondération séparés (facteurs de répartition) soient assignés pour la répartition du détriment entre les différents tissus de l'arbre respiratoire, comme indiqué dans le tableau II. 
INCERTITUDES SUR LA DOSE DÉLIVRÉE AUX VOIES RESPIRATOIRES...

\section{TABLEAU II}

Facteurs de pondération assignés pour la répartition du détriment radiologique dans les différents tissus pulmonaires d'après la publication 66 de la CIPR

(ICRP, 1994 b).

Weighting factors assigned for the partition of radiation detriment among respiratory tract tissues from ICRP publication 66 (ICRP, 1994 b).

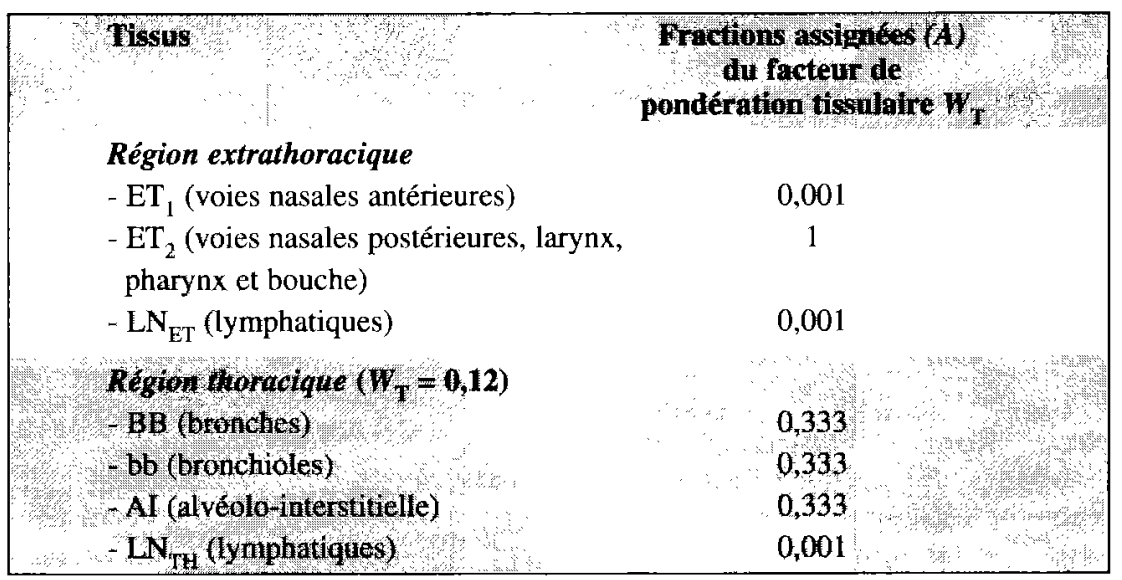

Quand on applique le nouveau modèle de la CIPR 66 (ICRP, 1994b) pour calculer la dose efficace par unité d'exposition dans les mines, la dose efficace par unité d'exposition qui en résulte est 2 à 3 fois supérieure à celle provenant des études épidémiologiques. Les estimations dosimétriques sont d'environ $15 \mathrm{mSv}$ par WLM, alors que les estimations épidémiologiques sont d'environ $5 \mathrm{mSv}$ par WLM (ICRP, 1994 a). Pour des conditions typiques d'exposition dans les mines, la valeur du coefficient de conversion de dose, $E / P_{\mathrm{p}}$, a été trouvée comme étant de $13,4 \mathrm{mSv}$ par WLM (ICRP, 1994b).

La $C I P R$ a résolu cette incompatibilité entre les deux approches en utilisant une autre méthode pour dériver $E / P_{\mathrm{p}}$. Premièrement, le nombre estimé de cancers du poumon en excès chez les mineurs par unité d'exposition, extrapolé directement des études épidémiologiques sur les mineurs d'uranium, est divisé par le nombre attendu de cancers du poumon en excès par unité de dose. Cette dernière quantité est extrapolée directement de l'incidence de cancers du poumon par unité de dose d'irradiation globale gamma obtenue à partir des données sur les survivants de Hiroshima et Nagasaki.

Cette méthode conduit à une convention de conversion de dose d'environ $5 \mathrm{mSv}$ par WLM pour les travailleurs, en supposant une durée du travail de 2000 heures par an et un facteur d'équilibre de 0,4 (ICRP, 1994a). Pour les membres du public, 
la convention de conversion correspondante est de 3,88 mSv, en supposant une durée d'exposition de 7000 heures par an dans l'habitat et un facteur d'équilibre de 0,4 . Ces conventions de conversion sont basées sur l'égalité du détriment radiologique et non sur la dosimétrie.

Une analyse d'incertitude sur la dose efficace par unité d'exposition aux descendants du radon a été réalisée par Birchall et James (Birchall and James, 1994) afin de déterminer si l'incertitude sur les valeurs des paramètres de l'approche dosimétrique pouvait expliquer les différences existant avec l'approche épidémiologique. Leur conclusion est que si les valeurs de 20 et 0,12 sont utilisées respectivement pour les facteurs de pondération dosimétrique, $W_{\mathrm{R}}$ et $W_{\mathrm{T}}$, il est improbable que la dose efficace par unité d'exposition aux descendants du radon, $E / P_{\mathrm{p}}$, dans une mine souterraine puisse être aussi faible que la valeur déterminée à partir des données épidémiologiques sur les mineurs d'uranium, c'est-àdire, $5 \mathrm{mSv}$ par WLM. Cette analyse suggère qu'il n'est pas vraisemblable que la différence d'un facteur trois qui existe entre l'évaluation dosimétrique et l'évaluation épidémiologique du risque puisse être expliquée uniquement par des incertitudes sur le calcul de la dose, c'est-à-dire, les conditions de l'aérosol, les paramètres du modèle pulmonaire ou les hypothèses sur les cellules cibles. Cette analyse implique qu'il peut y avoir des erreurs systématiques dans au moins une des valeurs recommandées par la $C I P R$ pour les facteurs de pondération $W_{\mathrm{T}}, W_{\mathrm{R}}$ ou DDREF. De plus, si l'on admet cette implication pour l'exposition aux descendants du radon, elle doit aussi être acceptée pour n'importe quels radionucléides émetteurs alpha tels que les isotopes du plutonium.

Différents éléments peuvent expliquer les incertitudes sur ces facteurs de pondération :

- la valeur du facteur de pondération du rayonnement alpha $W_{\mathrm{R}}=20$, est basée sur l'hypothèse d'une répartition homogène de l'énergie au niveau des tissus cible, ce qui n'est pas le cas des descendants du radon. Les dépôts d'énergie des descendants du radon inhalés dans les voies respiratoires ont un caractère stochastique (Hofmann, 1998). Par ailleurs les valeurs du rapport de la dose absorbée après exposition au radon et à ses descendants par comparaison à des expositions aux rayons gamma obtenues à partir de différentes études expérimentales conduit à des équivalents de dose absorbée en $\mathrm{mGy}_{\text {WLM }}{ }^{1}$ de plus en plus faibles (Cross and Monchaux, 1998). Ainsi, l'utilisation d'un facteur de pondération de 20 pour les rayonnements alpha aboutirait à une surestimestion du risque de l'exposition au radon et à ses descendants chez le rat. Il est probable qu'il en soit de même dans le cas des expositions humaines;

- le facteur de pondération tissulaire pour le poumon $W_{\mathrm{T}}=0,12$ est basé sur l'observation de la survenue des cancers chez les survivants de Hiroshima et Nagasaki qui ont subi une irradiation globale externe délivrée à fort débit dans un temps très court. Dans le cas des expositions au radon et ses descendants, 
on se trouve au contraire dans la situation d'une exposition interne par inhalation, conduisant à une irradiation hétérogène des tissus de l'arbre respiratoire. Dans le cas des cancers du poumon, le risque peut être considérablement augmenté du fait de l'exposition à d'autres cancérogènes, comme la fumée de cigarettes. Par ailleurs, la répartition régionale du risque stochastique dans les différentes régions de l'arbre respiratoire a été établie à partir des données sur les mineurs, de celles des survivants de Hiroshima et Nagasaki, d'études expérimentales chez l'animal ou sur des systèmes cellulaires. Ces paramètres sont susceptibles de révision qui entraîneraient des modifications de l'estimation du risque ;

- la valeur du facteur de pondération des effets du débit de dose DDREF = 2 a été fixée pour tenir compte de la probabilité de cancers pour les expositions à faible débit par comparaison avec la mortalité par cancer des survivants de Hiroshima et Nagasaki, exposés à fort débit. Dans le cas des expositions au radon, des études expérimentales récentes ont montré que pour des expositions cumulées relativement faibles, inférieures ou égales à $0,35 \mathrm{~J} \mathrm{~h} \mathrm{~m}^{-3}(100$ WLM), une diminution du débit d'exposition entraînait une diminution du risque de cancer du poumon chez le rat (Morlier et al., 1992, 1994; Monchaux et al., 1999). Il est possible que facteur qui intervient comme quotient dans l'estimation du risque soit sous-évalué dans le cas des expositions au radon.

\section{Comparaison entre les différents modèles dosimétriques et influence des variables de modélisation}

Au cours des 10-15 dernières années, tous les modèles dosimétriques sur le radon ont eu tendance à prédire une dose par unité d'exposition plus élevée que celle dérivée de l'épidémiologie. Les raisons de cette tendance sont que, en dépit de la complexité de la modélisation dosimétrique pulmonaire, les doses qui en résultent semblent assez solidement établies et insensibles à beaucoup des hypothèses utilisées.

Les estimations de dose obtenues en utilisant les divers modèles, pour différentes tailles de particules, différentes valeurs de « fraction libre » et de volumes respiratoires sont généralement en bon accord et peuvent varier d'un facteur d'environ 2 autour des valeurs normales de ces facteurs.

L'utilisation du modèle pulmonaire de la CIPR 66 (ICRP, 1994b) augmente les doses équivalentes pondérées au poumon par WLM $\left(H_{\mathrm{W}} / P_{\mathrm{p}}\right)$ d'un facteur d'environ 2 par comparaison avec les modèles de Jacobi-Eisfeld (Jacobi and Eisfeld, 1980) et de James-Birchall (James et al., 1980). La prise en compte des cellules sécrétoires dans la région bronchique (BB) augmente la dose dans cette région d'un facteur 1,6 et augmente l'équivalent de dose pulmonaire $H_{\mathrm{W}} / P_{\mathrm{p}}$ d'un 
facteur 1,2 (Marsh and Birchall, 1998b). De même, l'hypothèse utilisée par CIPR 66 (ICRP, 1994b), selon laquelle la fraction du facteur de pondération tissulaire (A) assignée à chacune des trois régions du poumon $\mathrm{BB}$, bb et $\mathrm{AI}$ est de 0,333 , équivaut à donner des valeurs de $\mathrm{A}$ de 0,666 pour la région trachéo-bronchique (TB) et de 0,333 pour la région pulmonaire, au lieu de 0,5 pour la région TB et de 0,5 pour la région pulmonaire selon la CIPR 32 (ICRP, 1981), augmentant ainsi la dose à la région TB d'un facteur de 1,3 (Marsh and Birchall, 1998b).

\section{Conclusion}

La relation entre l'exposition aux descendants à vie courte du radon dans l'air et les doses aux cellules considérées à risque pour l'induction de cancers de l'appareil respiratoire chez l'homme est extrêmement complexe. Il n'y a pas de conflits fondamentaux majeurs entre les différents modèles disponibles et le nouveau modèle pulmonaire de la CIPR (ICRP, 1994b). Pour la plupart des conditions d'exposition, tous les modèles conduisent à des valeurs de dose qui sont en accord avec des variations d'un facteur d'environ 2. Les différences qui existent entre les modèles proviennent surtout de l'identification des cellules cibles, du choix des modèles morphométriques, des modèles de dépôt et des paramètres physiologiques.

Des recherches complémentaires sont nécessaires pour réduire les incertitudes dans la modélisation dosimétrique pulmonaire. Les principales zones d'incertitude à examiner sont les distributions pondérées en taille de l'activité des descendants du radon sur les lieux de travail et dans les habitations, les modalités de dépôt dans l'arbre respiratoire (paramètres liés au sujet), l'identification des cellules à risque et leur importance en cancérogenèse pulmonaire (paramètres liés aux cellules cibles), le comportement physique des descendants du radon déposés dans le mucus ainsi que leurs taux d'absorption (taux de solubilité et de clairance), et les valeurs des facteurs de pondération du risque, $W_{\mathrm{T}}, W_{\mathrm{R}}$ et DDREF qui sont propres à l'irradiation alpha du poumon par les descendants du radon dans les situations professionnelles ou de l'environnement domestique.

Une meilleure caractérisation des paramètres qui régissent la pénétration des aérosols dans les voies aériennes, leurs taux de dépôt et de clairance devraient permettre d'établir une évaluation globale réaliste de l'incertitude sur l'établissement des facteurs de conversion exposition-dose, à la fois pour les travailleurs et pour le public. Une amélioration des informations sur ces données et la réduction des incertitudes sur la dose absorbée devraient permettre, à terme, de réduire l'écart entre les estimations du risque obtenues entre l'approche dosimétrique et l'approche épidémiologique. 


\section{RÉFÉRENCES}

Altschuler B., Nelson N., Kuschner M. (1964) Estimation of lung tissue dose from the inhalation of radon and daughters. Health Phys., 10, 1137-1161.

Andersson M., Wallin H., Jönsson M., Nielsen L.L., Visfeldt J., Vyberg M., Bennet W.P., De Benedetti V.M.G., Travis L.B., Storm H.H. (1995) Lung cancer and malignant mesothelioma in patients exposed to Thorotrast: incidence, histology and $p 53$ status. Int. J. Cancer, 63, 330-336.

Birchall A., James A.C. (1994) Uncertainty analysis of the effective dose per unit exposure from radon progeny and implications for ICRP risk-weighting factors. Radiat. Prot. Dosim., 53, 1-4, 133140 .

Booker D.V., Chamberlain A.C., Newton D., Stott A.N.B. (1969) Uptake of radioactive lead following inhalation and injection. Br. J. Radiol., 42, 457-466.

Cross F.T., Monchaux G. (1998) Bases expérimentales de l'évaluation du risque de cancer. In «Le radon», Collection IPSN, H. Métivier et M.C. Robé, Ed., (EDP Sciences, Les Ulis) pp. 125-153.

Darby S.C., Nakashima E., Kato H. (1985) A parallel analysis of cancer mortality among atomic bomb survivors and patients with ankylosing spondylitis given X-ray therapy. J. Natl Cancer Inst., 75, $1-21$.

Fisher D.R., Hui T.E., Bond V.P., James A.C. (1992) Microdosimetry of radon progeny: application to risk assessment. In : Indoor Radon and Lung Cancer : Reality or Myth ? Part 1. Proceedings of the 29th Hanford Symposium on Health and the Environment. F.T. Cross, Ed., pp. 307-321 Battelle Press, Columbus, Ohio.

Grampa G. (1971) Radiation injury with particular reference to Thorotrast. Pathobiol. Annu., 6, 147-169.

Harley N.H., Cohen B.S., Robbins E.S. (1996) The variability in radon decay product bronchial dose. Environment International, 22, Suppl. 1, S959-S964.

Harley N.H., Pasternack B.S. (1982) Environmental radon daughter alpha dose factors in a five-lobed human lung. Health Phys. 42, 789-799.

Hofmann W. (1998). Overview of radon lung dosimetry. Radiat. Protect. Dosim., 79, 229-236.

Horacek J., Placek V., Sevc J. (1977) Histologic types of bronchogenic cancer in relation to different conditions of radiation exposure. Cancer, 40, 832-835.

Hursh J.B., Mercer T.T. (1970) Measurement of ${ }^{212} \mathrm{~Pb}$ loss rate from human lungs. J. Appl. Physiol., 28, 268-274.

Hursh J.B., Schraub A., Sattler E.L., Hofmann H.P. (1969) Fate of ${ }^{212} \mathrm{~Pb}$ inhaled by human subjects. Health Phys., 16, 257-267.

ICRP (1979) Limits for Intakes of Radionuclides by Workers. ICRP Publication 30, Part 1. Pergamon, Oxford, UK.

ICRP (1981) Limits for Inhalation of Radon Daughters by Workers. ICRP Publication 32. Annals of the ICRP 6 (1). Pergamon, Oxford, UK.

ICRP (1987) Lung cancer risk from indoor exposutres to radon daughters. ICRP Publication 50. Pergamon, Oxford, UK.

ICRP (1990) Recommendations of the International Commission on Radiological Protection. ICRP Publication 60. (Annals of the ICRP Vol. 21 No. 1-3). Pergamon, Oxford, UK.

ICRP (1994a) Protection against radon-222 at home and at work. ICRP Publication 65. Pergamon, Oxford, UK.

ICRP (1994b) Human respiratory tract model for radiological protection. ICRP Publication 66. Pergamon, Oxford, UK.

Jacobi W. (1964) The dose to the human respiratory tract by inhalation of short-lived ${ }^{222} \mathrm{Rn}$ - and ${ }^{220} \mathrm{Rn}-$ decay products. Health Phys., 10, 1163-1174. 
Jacobi W., Eisfeld K. (1980) Dose to tissues and effective dose equivalent by inhalation of radon-222, radon-220 and their short-lived daughters. GSF Report S-626. Gesellschaft für Strahlen und Umweltforschung, Munich-Neuherberg, West Germany.

James A.C. (1988). Lung dosimetry. In: Radon and Its Decay Products in Indoor Air, pp. 259-309, W.W Nazaroff and A.V. Nero, Jr, eds., (John Wiley and Sons Inc., New York, USA).

James A.C., Greenhalgh J.R., Birchall A. (1980) A dosimetric model for tissues of the human respiratory tract at risk from inhaled radon and thoron daughters. In : Radiation Protection. A Systematic Approach to Safety, vol. 2., pp. 1045-1048. Pergamon, Oxford, UK.

Jasmin J.R., Bronchériou C., Morin M., Jasmin C. (1977) Modèles expérimentaux de cancers de la cavité buccale : les cancers radioinduits par le chlorure de cérium 144. Actual. Odontostomatol., 118, 289-298.

Johnson N.F., Hubbs A.F. (1990) Epithelial progenitor cells in the rat trachea. Am. J. respir. Cell. Mol. Biol., 3, 579-585.

Marsh J.W, Birchall A. (1998a) Determination of lung-to-blood absorption rates for lead and bismuth that are appropriate for radon progeny, Memorandum NRPB-M929, May 1998, NRPB, Chilton, Didcot, Oxfordshire, OX 11 ORQ, UK.

Marsh J.W., Birchall A. (1998b) Sensitivity analysis of the weighted equivalent lung dose per unit exposure from radon progeny, Memorandum NRPB-M915, June 1998, NRPB, Chilton, Didcot, Oxfordshire, OXI1 ORQ, UK.

Muscat J.E., Wynder E.L. (1995) Lung cancer pathology in smokers, ex-smokers and never smokers. Cancer Letters, 88, $1-5$.

Monchaux G., Morlier J.P., Altmeyer S., Debroche M., Morin M. (1999) Influence of exposure rate on lung cancer induction in rats exposed to radon progeny. Radiat. Res. (in press).

Morlier J.P., Morin M., Chameaud J., Masse R., Bottard S., Lafuma J. (1992) Importance du rôle du débit de dose sur l'apparition des cancers chez le rat après inhalation de radon. C.R. Acad. Sci., série III, 315, 463-466.

Morlier J.P., Morin M., Monchaux G., Pineau J.F., Chameaud J., Lafuma J., Masse R. (1994) Lung cancer incidence after exposure of rats to low doses of radon: influence of dose rate. Radiat. Protect. Dosim., 56, 93-97.

NCRP (1997) Deposition, Retention and Dosimetry of Inhaled Radioactive Substances. NCRP Report, $n^{\circ}$ 125. National Council on Radiation Protection and Measurements, Bethesda, MD, USA.

NRC (1990) Health Effects of Exposure to Ionizing Radiation: BEIR-V. National Research Council, National Academy Press, Washington, D.C., USA.

Phalen R.F., Oldham M.J., Beaucage C.B., Crocker, T.T., Mortensen J.D. (1985) Postnatal enlargement of human tracheobronchial airways and implications for particle deposition. Anat. Rec., 212, 368-380.

Roy M., Rannou A. (1998) Dosimétrie interne des expositions au radon. In «Le radon», Collection IPSN, Métivier H. et Robé M.C., eds, (EDP Sciences) pp. 155-183.

Rowland R.E. (1994) Radium in Humans. A Review of U.S. Studies. Argonne National Laboratory, September 1994, 9700 South Cass Avenue, Argonne, Illinois 60439, USA.

Saccomanno G., Archer V.E., Auerbach O., Kuschner M., Saunders R.P., Klein M.G. (1971) Histologic types of lung cancer among uranium miners. Cancer, 27, 515-523.

Saccomanno G., Archer V.E., Saunders R.P., James L.A., Beckler P.A. (1964) Lung cancers of uranium miners on the Colorado Plateau. Health Phys., 10, 1195-1201.

Saccomanno G., Archer V.E., Auerbach O., Kuschner M., Harley N.H., Michels R.Y., Anderson M.W., Bechtel J.J. (1996) A comparison between the localization of lung tumors in uranium miners and in nonminers from 1947 to 1991. Cancer, 77, 1278-1283.

Saccomanno G., Huth G.C., Auerbach O., Kuschner M. (1988) Relationship of radioactive radon daughters and cigarette smoking in the genesis of lung cancer in uranium miners. Cancer, 62, 1402-1408. 


\section{INCERTITUDES SUR LA DOSE DÉLIVRÉE AUX VOIES RESPIRATOIRES...}

Thun M.J., Lally C.A., Flannery J.T., Calle E.E., Flanders W.D., Heath C.W. Jr. (1997) Cigarette smoking and changes in the histopathology of lung cancer. J. Natl Cancer Inst., 89, 1580-1586.

TGLD (Task Group on Lung Dynamics) (1966) Deposition and retention models for internal dosimetry of the human respiratory tract. Health Phys., 12, 173-207.

UNSCEAR (1988) Sources, effects and risks from ionizing radiation. United Nations Scientific Committee on the Effects of Atomic Radiations. Report of General Assembly with Scientific Annexes: Publication $\mathrm{n}^{\circ} \mathrm{E}$ 88.IX.7, 1988, ONU, New-York.

Weibel E.R. (1963), Morphometry of the Human Lung. Academic Press, New York, USA.

Wynder E.L., Muscat J.E. (1995) The changing epidemiology of smoking and lung cancer histology. Environ. Health Perspect., 103 (Suppl.8), 143-148.

Yeh H.C., Schum G.M. (1980) Models of human airways and their application to inhaled particle deposition. Bull. Math. Biol., 42, 461-480.

Yessner R. (1981) The dynamic histopathologic spectrum of lung cancer. Yale J. Biol. Med., 54, 447-756. 